\title{
On the Physics Description of Fusion Plasmas I
}

\author{
Jan Weiland ${ }^{\mathrm{a}}$, Anatoly Zagorodny ${ }^{\mathrm{b}}$, Vlodymyr Zasenko ${ }^{\mathrm{b}}$ and Nana \\ Shatashvilic \\ ${ }^{a}$ Chalmers University of Technology, S-41296 Gothenburg, Sweden \\ ${ }^{b}$ Bogoliubov Institute for Theoretical Physics, 03680 Kiev, Ukraine \\ ${ }^{c}$ Ivane Javahishvili Tbilisi State University, Tbilisi 028, Georgia, and \\ TSU Andronikashvili Institute of Physics, Department of Plasma Physics, Tbilisi,0177, Georgia
}

\begin{abstract}
The one fluid, two fluid and kinetic descriptions of fusion plasmas are discussed from basic theoretical points and from that of application to present day problems of relevance to fusion reactors. In particular both statistical and deterministic aspects are considered. Of special importance are non-Markovian effects.
\end{abstract}

Keywords: Fusion plasmas, fluid description, kinetic description

PACS: $52.35-\mathrm{g}, 52.35 . \mathrm{Kt}$

\section{FUSION PLASMAS}

There are essentially two types of plasmas considered relevant for nuclear fusion. These are magnetized low density plasmas and unmagnetized high density plasmas. In the first case confinement is due to the magnetic field while, in the second case, confinement is due to inertia. We will here focus on magnetized plasmas1-24. In order to reach conditions for thermonuclear fusion a plasma must have a temperature of at least 100 million degrees Celsius. At the same time the maximum average beta (ratio of plasma to magnetic field pressure)

$$
\beta=\frac{n T}{\mathrm{~B}^{2} / 2 \mu_{0}}
$$

which can be sustained is only a few \%. Thus we realize already on this ground that the density has to be quite low. Then there is also a direct limitation on density (Greenwald limit) that limits density further in the edge region. As it turns out, the pressure in a fusion plasma will be very similar to the atmospheric pressure. Thus with a temperature of 108 centigrades we realize that the density will be quite low. A typical property of low density, high temperature plasmas is that close collisions between particles will be very rare. Instead the dynamics will be dominated by collective effects. As we expect, collective effects, as well as close collisions, will try to destroy the magnetic confinement. This is in agreement with the general laws of thermodynamics. A simple way of looking at global, electromagnetic, instabilities 1,-5 is that the plasma sets up currents that generate magnetic fields that, in turn, create a less efficient magnetic bottle. Such instabilities are driven by current gradients. There

Joint ITER-IAEA-ICTP Advanced Workshop on Fusion and Plasma Physics

AIP Conf. Proc. 1445, 34-53 (2012); doi: 10.1063/1.3701884

(C) 2012 American Institute of Physics 978-0-7354-1041-1/\$30.00 
will also be instabilities driven by density and temperature gradients. All these gradients are examples of gradients that we need to maintain in order to confine the plasma for such a long time that we get more energy out than we put in.

\section{STABILITY AND TRANSPORT}

Global stability, as indicated in the previous section, is of outmost importance to a magnetic confinement system. The main stabilizing effect of such instabilities is the bending of magnetic field lines associated with Alfvén waves ${ }^{1}$. A quite general energy principle for global stability, containing the stabilization by line bending, was then derived $^{2}$. Confinement is usually divided into stability and transport where stability then means stability to the very fast global instabilities of a Magnetohydrodynamic (MHD) type (mainly associated to current or pressure gradients) while transport refers to the transport due to more localized (micro) instabilities that drive fluxes of a magnitude that does not prevent a steady state where the confinement time is sufficiently long for a positive energy balance. Of course there can also be intermediate cases ${ }^{4,5}$. However, independently of if we are considering global or micro instabilities, the most dangerous modes are those where the real eigenfrequency is much smaller than the growthrate. We are mainly interested in low frequency phenomena, $\omega<<\Omega_{\mathrm{ci}}$ (this applies to MHD and transport, not to heating).

Lower frequency perturbations give more transport!

$$
\chi_{i}=\frac{\gamma^{3} / k_{r}^{2}}{\omega_{r}^{2}+\gamma^{2}}
$$

The non-Markovian type of diffusivity shown by (1.2) was first derived by fluid theory ${ }^{6}$ but more recently ${ }^{7}$ also by using a kinetic orbit integration technique. The real frequency here represents a coherent periodic motion which reduces transport. Clearly transport is a nonperiodic secular phenomenon which is due to the decorrelation by the growthrate. It is actually the linear growthrate that enters here in a nonlinear steady state since it represents the source of the turbulence ${ }^{9}$. We would, however, argue that we should use only the part of the linear growth rate that is due to the inhomogeneities of the quantities we study the transport of, i.e. we should have a self consistent relaxation!

\section{THERMODYNAMICS}

Since our aim is to confine a plasma we cannot avoid to have gradients. However, gradients mean that the system is not in thermodynamic equilibrium. Thus there will always be free energy available that potentially can drive instabilities. Since we primarily want to confine density and temperature, instabilities driven by gradients in these are what we should look for. As it turns out, the relaxations of density and temperature are coupled. Thus we have to use a full transport matrix. The main trend is that the relaxations of density and temperature are competing in such a way that the equilibrium will have comparable length scales ${ }^{5,18}$ of density and temperature when 
the sources are comparable. Of course we can create a situation where the temperature is more peaked than the density by having a much stronger central heating than fuelling. This would, however create a particle pinch $^{18}$ that would reduce the difference in length scales considerably.

\section{DIFFERENT TYPES OF PHYSICS DESCRIPTIONS}

With physics description we generally mean one fluid, multi fluid or kinetic descriptions

The one fluid description is usually used for describing MHD instabilities

MHD instabilities generally require detailed geometry. However, their growthrate is so large that it is typically larger than the drift frequencies (which are different for different species) and, accordingly, we can use a single fluid description.

Microinstabilities are more localized. They can often be described by a WKB approximation and are thus not quite so sensitive to geometry. On the other hand growthrates are of the order of drift frequencies which are different for different species. Thus multifluid or kinetic descriptions are needed.

Toroidal effects represent the third dimension in which particles are not confined by the magnetic field. Although microinstabilities are less sensitive to geometry, toroidal effects are very important, in particular in the core.

\section{THE USE OF DIFFERENT DESCRIPTIONS}

A very strong fusion community dealing primarily with large scale instabilities (which are indeed the most dangerous) has made single fluid MHD equations one of the most used descriptions. Unfortunately the difficulties with dealing with more detailed two fluid effects and the success of one fluid equations outside of their formal regime of applicability has led to a too strong focus on one fluid equations, some researcher using only one fluid or kinetic descriptions. As it turns out, a multi fluid description is usually the best for all types of low frequency modes once you know how to deal with convective diamagnetic and stress tensor effects. This is so since the two fluid derivation of MHD type modes is not much more complicated than the one fluid derivation and advanced fluid closures make a kinetic treatment unnecessary for instabilities driven by gradients in configuration space (this excludes modes driven resonantly by fast particles). Unfortunately there are also risks of making mistakes with e.g. convective diamagnetic effects and this has sometimes lead researchers to avoid multi fluid descriptions. In many cases also small ion temperature has been assumed. In this way it is possible to avoid most of the difficulties with the two fluid approach. However, one of the main goals with fusion plasmas is to get sufficiently high ion temperature for thermonuclear reactions! More recently the use of gyrokinetic descriptions has taken over in cases with reasonable simple calculations. In this case kinetic descriptions are often considered to be more "first principles". Thus it is important to define what we mean by "first principles". The original meaning is that we should start with some basic description of the physics and make derivations until we obtain a model which is suitable for our intended study. The

original meaning of "first principles" is as opposed to empirical. Thus a "first 
principles" transport model must not use inputs from experiment. Concerning kinetic models, it is important to note that not even these have a general, unquestionable, validity ${ }^{10}$. Thus we would prefer to reserve the term "first principles" for proceedures that start from a basic theoretical description and derives a model without making use of empirical facts.

Some outstanding research was made in the 1970's with the Hasegawa - Mima equation by using small ion temperature ${ }^{11}$. This research reviled the cascades both to lower and higher modenumbers and the generation of zonal flows. However, including ion temperature effects requires a description with several similar equations and today further work with small ion temperature equations are mainly mathematical exercises.

\section{FLUID DESCRIPTION}

When fluid equations are derived from kinetic equations we end up with multi fluid equations. The simplest case of these are the two fluid equations including only electrons and one ion species. The generalization to multiple ion species is straight forward so it is convenient to make most of the multi fluid derivations for the simple two fluid case. This is, however only from the conceptual point of view. Effects of impurities are usually important in fusion plasmas. We will here start from the two fluid model.

$$
\begin{aligned}
\frac{\partial \mathbf{v}}{\partial t}+ & (\mathbf{v} \cdot \nabla) \mathbf{v}=\frac{q}{m}(\mathbf{E}+\mathbf{v} \times \mathbf{B})-\frac{1}{m n}(\nabla P+\nabla \cdot \boldsymbol{\pi})=0 \\
\omega & <<\Omega_{c} \Rightarrow \\
\mathbf{v}_{\perp} & =\mathbf{v}_{E}+\mathbf{v}_{p}+\mathbf{v}_{*} \\
\mathbf{v}_{E} & =\frac{1}{B}(\mathbf{E} \times \widehat{\mathbf{z}}) \quad(\text { ExB drift }) \\
\mathbf{v}_{*} & =\frac{\widehat{\mathbf{z}} \times \nabla P}{\mathrm{qnB}} \quad \text { (diamagnetic drift) } \\
\mathbf{v}_{p} & =\frac{1}{\Omega_{c}} \frac{\partial}{\partial t}(\widehat{\mathbf{z}} \times \mathbf{v}) \approx \frac{1}{B \Omega_{c}} \frac{\partial \mathbf{E}}{\partial \mathrm{t}} \quad \text { (polarization drift only kept for ions) }
\end{aligned}
$$

Here the diamagnetic drift is a pure fluid drift, i.e. it does not move particles.

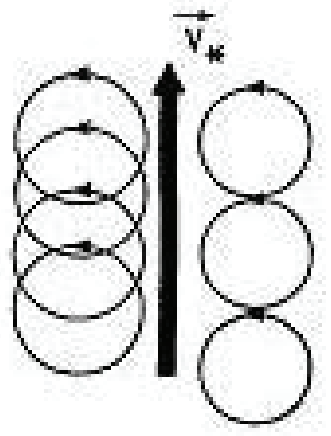

FIGURE 1. Diamagnetic drift 
Since the diamagnetic drift does not move particles it does not cause a perturbation in density, i.e.

$$
\nabla \cdot\left(\mathrm{n} \mathbf{v}_{*}\right)=0
$$

However, (1.4) is only valid for homogeneous magnetic field. We notice that there is no magnetic drift included in (1.3). This is because it is a pure particle drift (not a fluid drift). We will soon return to this case.

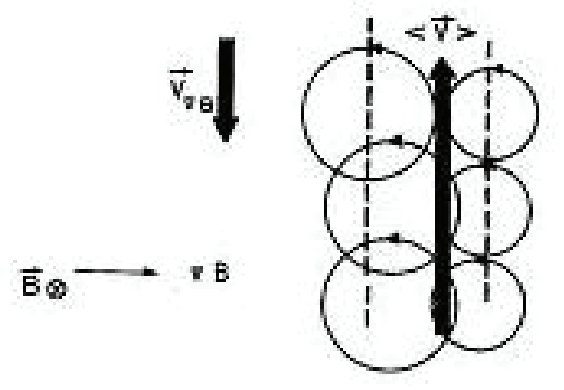

FIGURE 2. Magnetic drift. The particle drift is compensated by the fact that more particles contribute from the side with weaker magnetic field in such a way that there is no fluid drift.

$\mathrm{Eq}(1.4)$ is the lowest order consequence of the fact that the diamagnetic drift does not move particles. In the momentum equation the stress tensor cancel convective diamagnetic effects. Such effects are cancelled also in the energy equation as we will soon see.

\section{ALFVÉN WAVES}

As an example of a simple two fluid derivation of an MHD mode we will now show the derivation of Alfvén waves.

$$
\nabla \cdot \mathbf{j}=0
$$

(quasineutrality)

Since $\nabla \cdot\left(n \mathbf{v}_{*}\right)=0$ and the ExB drifts are equal we get:

$$
\nabla \cdot\left(n \mathbf{v}_{\mathbf{p}}\right)=-\nabla \cdot \mathrm{j}_{\|}=\frac{1}{\mu_{0}} \Delta \mathrm{A}_{\|}
$$

where we used $\delta B=\nabla x \mathrm{~A}_{\|} \widehat{\mathbf{z}}$

Now the MHD constraint $E_{\|}=0 \quad$ or $\quad \mathrm{A}_{\|}=\frac{1}{\omega} \phi$ 
can be used to express (1.5) in only the potential. We then get the dispersion relation for Alfvén waves ${ }^{1}$ :

$$
\omega^{2}=\mathrm{k}_{\|}^{2} \mathrm{v}_{\mathrm{A}}^{2}
$$

where $\quad \mathrm{v}_{\mathrm{A}}=\frac{\mathrm{B}_{0}}{\sqrt{\mu_{0} \mathrm{~nm}_{\mathrm{i}}}}$ is the Alfvén velocity.

This derivation is probably simpler than with the one with fluid equations.

\section{INTERCHANGE MODES}

If we now add magnetic curvature we have:

$$
\nabla \cdot\left(\mathrm{n} \mathbf{v}_{* j}\right)=\frac{1}{T} \mathbf{v}_{\mathbf{D}_{\mathbf{j}}} \cdot \nabla P_{j}
$$

This means that in a curved magnetic field, the diamagnetic drift also "contains" the magnetic drift in some sense.

Where $\mathrm{j}$ indicates particle species and $\mathbf{v}_{\mathbf{D}}$ is the magnetic drift Now, since

$$
\begin{aligned}
& \text { We get } \mathbf{v}_{D e}=-\frac{\mathrm{T}_{\mathrm{e}}}{\mathrm{T}_{i}} \mathbf{v}_{\mathbf{D} i} \\
& \nabla \cdot\left(\mathrm{n} \mathbf{v}_{* i}-\mathrm{n} \mathbf{v}_{* \mathbf{e}}\right)=\frac{1}{T_{i}} \mathbf{v}_{\mathbf{D} i} \cdot \nabla\left(\delta P_{i}+\delta P_{e}\right)
\end{aligned}
$$

The critical point of the physics description is here how we treat the pressure perturbations.

In single fluid MHD it is here conventional to use an adiabatic incompressional approximation. Thus we use only the convective perturbation:

$$
\delta \mathrm{P}_{\mathrm{j}}=-\xi \cdot \nabla \mathrm{P}_{\mathrm{j}}
$$

Where $\xi$ is the ExB displacement: $\quad \frac{\partial \xi}{\partial t}=\mathbf{v}_{\mathbf{E}}$

Since $\mathbf{v}_{\mathbf{D i}}$ is proportional to $\mathrm{T}_{\mathrm{i}}$ we notice that (1.8) only depends on temperatures through the pressures. This is a particular property of the ideal MHD limit. We can now write the total dispersion relation as ${ }^{5}$

$$
\omega^{2}=\mathrm{k}_{\|}^{2} \mathrm{v}_{\mathrm{A}}^{2}+\frac{\partial P}{P \partial r} \frac{\mathrm{T}_{\mathrm{e}}+\mathrm{T}_{\mathrm{i}}}{\mathrm{m}_{\mathrm{i}} \mathrm{R}_{\mathrm{c}}}
$$


Where $\mathrm{P}=\mathrm{P}_{\mathrm{e}}+\mathrm{P}_{\mathrm{i}}$. Of course also (8) depends on temperature only through the sum of ion and electron temperatures. Since we use quasineutrality for these low frequency perturbations, the density $\mathrm{n}$ is just a multiplicative factor. We can say that the ideal MHD limit is degenerate with respect to particle species regarding temperatures and pressures. $\mathrm{Eq}(6)$ is the dispersion relation for electromagnetic interchange modes where the last term is destabilizing (pressure typically decreases with $r$ ). In tokamak geometry we have to use an eigenvalue equation since both parallel modenumber and the curvature are space dependent. The curvature is destabilizing only on the outside of a tokamak so unstable modes tend to localise there and lead to ballooning like perturbations. Thus Eq (1.10) turns into the eigenvalue equation of the MHD ballooning mode in proper geometry.

\section{NONADIABATIC NONISOTHERMAL COMPRESSIONAL TWO FLUID EQUATIONS.}

As mentioned above the critical approximation, leading to the conventional treatment of ballooning modes in the MHD limit is (1.9). We will now extend this to a nonadiabatic compressional treatment. We use the energy equation:

$$
\frac{3}{2}\left(\frac{\partial}{\partial t}+\mathbf{v}_{i} \cdot \nabla\right) \mathrm{T}_{\mathrm{i}}+\mathrm{P}_{\mathrm{i}} \nabla \cdot \mathbf{v}_{i}=-\nabla \mathbf{q}_{* i}
$$

where

$$
\mathbf{q}_{* i}=\frac{5}{2} \frac{\mathrm{P}_{\mathrm{i}}}{\mathrm{m}_{\mathrm{i}} \Omega_{\mathrm{c}_{\mathrm{i}}}}\left(\mathbf{e}_{\|} x \nabla \mathrm{T}_{\mathrm{i}}\right) \quad \text { is the diamagnetic heat flow. }
$$

It fulfills

$$
\nabla \cdot \mathbf{q}_{* i}=-\frac{5}{2} \mathrm{nv}_{* \mathrm{i}} \cdot \nabla \mathrm{T}_{\mathrm{i}}+\frac{5}{2} \mathrm{n} \mathbf{v}_{\mathbf{D i}} \cdot \nabla \mathrm{T}_{\mathrm{i}}
$$

Here q. is the diamagnetic heatflow according to Braghinskii. Braghinskii obtained this in a collision dominated situation. It is, however possible to obtain q. also by taking the limit of isotropic temperatures from a collisionless derivation where the irreducible part of the fourth moment is ignored.

In (1.12) the first term on the right hand side is a convective diamagnetic term. Since diamagnetic drifts do not convect plasma such terms have to be cancelled in some way. Using the continuity equation in the form:

$$
\nabla \cdot \mathbf{v}_{\mathbf{i}}=-\frac{1}{\mathrm{n}}\left(\frac{\partial}{\partial t}+\mathbf{v} \cdot \nabla\right) \delta \mathrm{n}
$$

In (1.11) we find that the convective diamagnetic terms indeed cancel. We then get: 


$$
\frac{\delta T_{i}}{T_{i}}=\frac{\omega}{\omega-\frac{5}{3} \omega_{D_{i}}}\left[\frac{2}{3} \frac{\delta n_{i}}{n_{i}}-\frac{\omega_{*_{e}}}{\omega}\left(\frac{2}{3}-\eta_{i}\right) \frac{e \phi}{T_{e}}\right]
$$

Where

$$
\eta_{i}=\frac{L_{n}}{L_{T_{i}}} \quad L_{j}=-\frac{1}{j} \frac{\partial j}{\partial r} ; j=\mathrm{n}, \mathrm{T}_{\mathrm{j}}
$$

In a proper two fluid model, we also include the lowest order Finite Larmor Radius (FLR) effect. It enters through the polarization and stress tensor drifts.

Where now : $\quad \mathbf{v}=\mathbf{v}_{\mathbf{E}}+\mathbf{v}_{*}$

$$
\mathbf{v}_{p}=\frac{1}{\Omega_{c}}\left(\frac{\partial}{\partial t}+\mathbf{v} \cdot \nabla\right)(\widehat{\mathbf{z}} \times \mathbf{v})
$$

The stress tensor drift is: $\quad \mathbf{v}_{\Pi}=\frac{\widehat{\mathbf{z}} \times \nabla \cdot \Pi}{\mathrm{qnB}}$

After rather lengthy calculations, using a convective density perturbation one arrives at:

$$
\nabla \cdot\left[\mathrm{n}\left(\mathbf{v}_{\mathbf{p}_{\mathrm{i}}}+\mathbf{v}_{\Pi_{\mathrm{i}}}\right)\right]=-i \mathrm{nk}^{2} \rho_{\mathrm{s}}^{2}\left(\omega-\omega_{* i T}\right) \frac{e \phi}{\mathrm{T}_{\mathrm{e}}}
$$

Where the diamagnetic drift with subindex $\mathrm{T}$ includes the full pressure gradient. It appears as a convective diamagnetic effect but comes from ExB convective density and temperature perturbations. Such perturbations are here substituted because the FLR term is assumed to be small but have, in practice, turned out to work better than expected. This is seen from a comparison with gyrokinetics.

\section{THE PARALLEL ELECTRIC FIELD}

We have already used the MHD limit $\mathbf{E} \cdot \mathbf{B}=0$ in our derivation of Alfvén waves. We will now see under which circumstances this is a good approximation. For this purpose it is useful to combine the parallel equation of motion of electrons (Ohms law) with the electron continuity equation. Ignoring electron inertia we get:

where

$$
\frac{\delta n_{e f}}{n_{e f}}=\frac{e}{T_{e}}\left(\phi-\frac{\omega-\omega_{* e T}}{k_{\|}} A_{\|}\right)-\frac{\delta T_{e}}{T_{e}}+i \frac{\mathrm{v}_{\| \mathrm{e}}}{k_{\|} D_{e}}
$$

$$
D_{e}=\frac{T_{e}}{m_{e} v_{e}}
$$

We now need an equation of state for electrons. At these low frequencies electrons are isothermal. However they thermalize along magnetic field lines that are bent. Thus we get: 


$$
\delta T_{e}=\eta_{\mathrm{e}} \frac{\omega_{* e}}{k_{\|}} e \mathrm{~A}_{\|}
$$

This is just radial convection in the background temperature gradient due to the bending of the fieldline.

We then get:

$$
\frac{\delta n_{e f}}{n_{e f}}=\frac{e}{T_{e}}\left(\phi-\frac{\omega-\omega_{* e}}{k_{\|}} A_{\|}\right)+i \frac{\mathrm{v}_{\| \mathrm{e}}}{k_{\|} D_{e}}
$$

Thus the electron temperature gradient parts cancel exactly.

The electron continuity equation is:

$$
\frac{\partial n_{e f}}{\partial t}+\nabla \cdot\left[n_{e f}\left(\mathbf{v}_{\mathbf{E}}+\mathbf{v}_{* \mathrm{e}}+\mathrm{V}_{\| 0} \frac{\delta \mathbf{B}_{\perp}}{\mathrm{B}}+\mathrm{v}_{\|} \widehat{\mathbf{e}}_{\|}\right)\right]=0
$$

Here we included a background parallel electron motion, giving a radial motion due to the bending of the fieldlines. This will include the Kink term.

Now using also

$$
\frac{\delta \mathbf{B}_{\perp}}{\mathrm{B}} \cdot \nabla \mathbf{J}_{\| 0}=\frac{d \mathbf{J}_{\| 0}}{\mathrm{dr}} \frac{1}{\mathrm{~B} r} \frac{\partial A_{\|}}{\partial \theta}
$$

We arrive at the relation between electrostatic and electromagnetic potentials

$$
\frac{e A_{\|}}{T_{e}}=k_{\|} \frac{\omega-\omega_{* e}}{\omega\left(\omega-\omega_{* e}\right)+\omega_{D e}\left(\omega_{* e T}-\omega\right)-\frac{k_{\|} k_{\theta} T_{e}}{e^{2} B n_{0}} \frac{\partial \mathbf{J}_{\| 0}}{\partial \mathrm{r}}-\mathrm{k}_{\perp}{ }^{2} \rho_{s}{ }^{2} k_{\|}{ }^{2} \mathrm{v}_{\mathrm{A}}{ }^{2}(1-i \delta)} \frac{e \phi}{T_{e}}
$$

where

$$
\delta=\frac{\omega-\omega_{D e}}{k_{\|}^{2} D_{e}}
$$

Then

$$
E_{\|}=k_{\|} \phi-\omega A_{\|}=k_{\|} \phi \frac{\omega_{D_{e}}\left(\omega_{* e T}-\omega\right)-\mathrm{k}_{\perp}{ }^{2} \rho_{s}{ }^{2} k_{\|}{ }^{2} \mathrm{v}_{\mathrm{A}}{ }^{2}-\frac{k_{\|} k_{\theta} T_{e}}{e^{2} B n_{0}} \frac{\partial \mathbf{J}_{\| 0}}{\partial \mathrm{r}}}{\omega\left(\omega-\omega_{* e}\right)+\omega_{D e}\left(\omega_{* e T}-\omega\right)-\frac{k_{\|} k_{\theta} T_{e}}{e^{2} B n_{0}} \frac{\partial \mathbf{J}_{\| 0}}{\partial \mathrm{r}}-\mathrm{k}_{\perp}{ }^{2} \rho_{s}{ }^{2} k_{\|}{ }^{2} \mathrm{v}_{\mathrm{A}}{ }^{2}(1-i \delta)}
$$

We note that FLR, curvature and current gradient contribute to a parallel electric field. Since the denominator is quadratic in $\omega$ and it contains the growthrate, which is larger than all drifts in ideal MHD we can see that the parallel electric field gets small in this limit. When the frequency is much smaller than the Alfvén frequency and FLR is not too small, the Alfvén terms dominate in both numerator and denominator and the broken rational factor approaches 1 . This is the electrostatic limit. The current 
gradient term also enters in (1.10) and gives the usual MHD kink mode by assuming vanishing parallel electric field. It enters mainly for low modenumbers and will usually be neglected in the following.

\section{DEGENERACY OF DENSITY AND TEMPERATURE GRADIENTS}

The linear gyrokinetic equation is:

$$
\left(\omega-\omega_{D}\left(\mathrm{v}_{\|}^{2}, \mathrm{v}_{\perp}^{2}\right)-k_{\|} \mathrm{v}_{\|}\right)\left(f_{k, \omega}^{(1)}+\frac{q \phi_{k, \omega}}{T} f_{0}\right)=\left(\omega-\omega_{\bullet}\right) \frac{q}{T} \phi_{k, \omega} e^{i L_{k}} J_{0}\left(\xi_{k}\right) f_{0}
$$

Leading to the density perturbation in $2 \mathrm{~d}$ (no parallel ion motion):

$$
\frac{\delta \mathrm{n}_{\mathrm{i}}}{\mathrm{n}_{\mathrm{i}}}=-\frac{e \phi}{T_{i}}\left[1-\frac{1}{n_{0}} \int_{0}^{\infty} \frac{\omega-\omega_{* i}\left[1+\eta_{i}\left(m_{i} \mathrm{v}^{2} / 2 T_{i}-3 / 2\right]\right.}{\omega-\omega_{D_{i}}\left(\mathrm{v}_{\|}^{2}+\mathrm{v}_{\perp}^{2} / 2\right) / \mathrm{v}_{\mathrm{th}}^{2}} J_{0}(\xi)^{2} f_{0} d^{3} \mathrm{v}\right]
$$

An expansion in FLR and $\omega_{\mathrm{D}} / \omega *$ up to quadratic terms (which is only allowed at the edge) gives:

$$
\frac{\delta n}{n}=\left[\frac{\omega_{* e}}{\omega}-\left(1-\frac{\omega_{* i T}}{\omega}\right)\left(1+\Gamma \frac{\omega_{D i}}{\omega}\right)\left(\frac{\omega_{D e}}{\omega}+k_{\perp}{ }^{2} \rho^{2}\right)+\Gamma \eta_{i} \frac{\omega_{* i} \omega_{D i} \omega_{D e}}{\omega^{3}}\right] \frac{e \phi}{T_{e}}
$$

where $\Gamma=7 / 4$. The corresponding fluid expansion, using (1.12) and (1.13), agrees except for that $\Gamma=5 / 3$. The difference is only $5 \%$ and seems to be due to that the kinetic temperature perturbations not are isotropic. We here notice the presence of $\eta_{\mathrm{i}}$ in the last term. This is clearly a term that can not be recovered from ideal MHD. It actually is the only nonadiabatic term here and is due to the diamagnetic heatflow in our two fluid model. For electromagnetic ballooning modes, this term gives us the kinetic ballooning mode. This mode is causing the difference to the MHD stability limits in Fig 3.

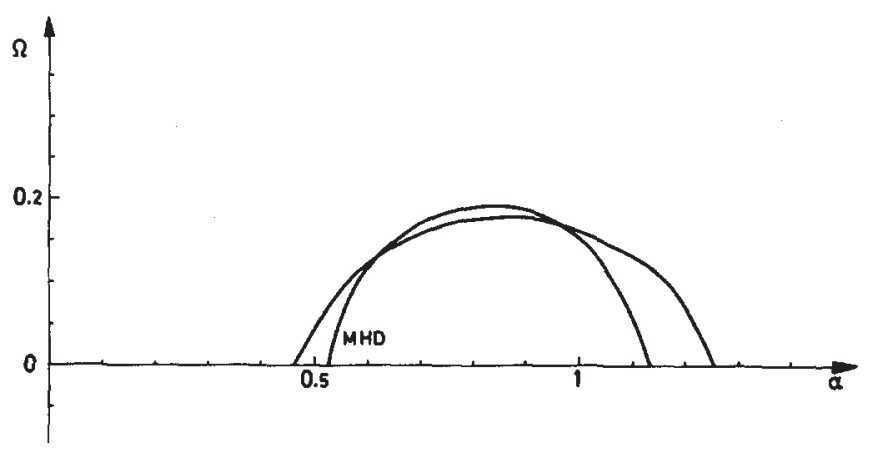

FIGURE 3 Growthrates of electromagnetic ballooning modes as a function of normalized $\beta$. The inner curve corresponds to ideal MHD while the outer includes Kinetic ballooning modes with a larger unstable region. Here $\varepsilon \mathrm{n}=0.35$, $\eta \mathrm{i}=2$ and $\mathrm{k} 2 \rho 2=0.01$. 
Although the effect of the genuine 2 fluid terms is rather modest here, it will be lager for larger $\eta_{\mathrm{i}}$ and $\varepsilon_{\mathrm{n}}$ and it was one of the main effects (together with peeling modes) that limited the slope of the H-mode barrier in recent simulations I have made. However, as we will see, using a single pressure gradient gives a dramatically worse approximation for Ion Temperature Gradient (ITG) modes.

One fluid limit for ITG modes

We write the continuity equation for ions:

$$
\frac{\partial \delta \mathrm{n}_{\mathrm{i}}}{\partial t}+\mathbf{v}_{\mathbf{E}} \cdot \nabla \mathrm{n}_{0}+\mathrm{n}_{0} \nabla \cdot \mathbf{v}_{\mathbf{E}}+\nabla \cdot\left(\mathrm{nv}_{* i}\right)=0
$$

Using (1.7) we get:

Now using (1.9) in the form

$$
\omega \delta \mathrm{n}_{\mathrm{i}}=\mathrm{n}_{0}\left(\omega_{*_{e}}-\omega_{D e}\right) \frac{e \phi}{T_{e}}-\frac{1}{T_{i}} \omega_{D i} \delta \mathrm{p}_{\mathrm{i}}
$$

$$
\frac{\delta \mathrm{p}_{\mathrm{i}}}{\mathrm{P}_{\mathrm{i}}}=\frac{\omega_{* \mathrm{i}}}{\omega} \frac{e \phi}{T_{i}}=\frac{\omega_{* \mathrm{i}}\left(1+\eta_{i}\right)}{\omega} \frac{e \phi}{T_{i}}
$$

and Boltzmann electrons with quasineutrality we get the dispersion relation:

$$
\omega^{2}-\omega\left(\omega_{* e}-\omega_{D e}\right)=-\tau \omega_{D i} \omega_{* \mathrm{i}}\left(1+\eta_{i}\right)
$$

With solution

$$
\omega=\frac{1}{2}\left(\omega_{* e}-\omega_{D e}\right)+\sqrt{\frac{1}{4}\left(\omega_{* e}-\omega_{D e}\right)^{2}-\tau \omega_{D i} \omega_{*_{i}}\left(1+\eta_{i}\right)}
$$

Here the stabilizing fist term under the root is often small due to the factor $1 / 4$ and, as it turns out, resistive instability would persist also if that term has stabilized the system. Thus the sign of the last term was sometimes given as the stability condition i.e. we get the stability limit:

$$
\eta_{i}=-1
$$

This result is, of course, completely wrong. It can be seen as a spurious result of an expansion in

$$
\frac{\omega_{D}}{\omega}<<1
$$

Actually, if we use the convective perturbation only for temperature we get 


$$
\begin{gathered}
\frac{\delta \mathrm{T}_{\mathrm{i}}}{\mathrm{T}_{\mathrm{i}}}=\eta_{i} \frac{\omega_{* \mathrm{i}}}{\omega} \frac{e \phi}{T_{i}} \\
\frac{\delta \mathrm{n}_{\mathrm{i}}}{\mathrm{n}_{\mathrm{i}}}=\frac{\omega_{* e}-\omega_{D e}-\tau \omega_{D i} \frac{\eta_{i} \omega_{* i}}{\omega} \frac{\delta \mathrm{T}_{\mathrm{i}}}{\mathrm{T}_{\mathrm{i}}} \frac{e \phi}{T_{e}}}{\omega-\omega_{D i}}
\end{gathered}
$$

We notice that the quadratic term in $\omega$ needed for instability vanishes for vanishing temperature gradient if (1.29) is combined with Boltzmann electrons. However such a term can be obtained by expansion in $\omega_{\mathrm{D}} / \omega$ ! This connects in a nice way to the common problem of expansion in $\omega_{\mathrm{D}} / \omega$ which was routinely done until the end of the 1980's.

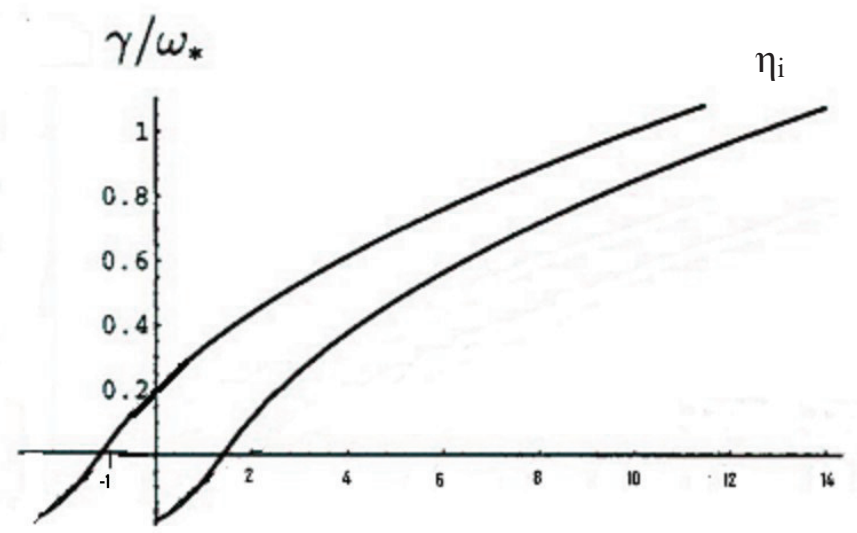

FIGURE 4. ITG threshold (-1) with convective pressure perturbation The correct threshold is shown by the right curve.

We note the very much larger error by using a convective pressure perturbation for the driftwave as compared to the electromagnetic ballooning mode (MHD type). Thus the drift wave is more sensitive to the physics description. We notice the upper stability regime in Fig 3. This is due to the magnetic field geometry. However, the effect of broken degeneracy with respect to temperature gradient is moderate. For the drift wave, however, there is no upper stability regime but the effect of broken degeneracy in temperature gradient is large. These two cases are examples of a general principle.

For MHD-type modes: Geometry is more important than the physics description For drift waves : The physics description is more important than geometry.

Nevertheless there are cases, in particular in enhanced confinement regimes where geometry can be quite important for drift waves. However, the very fact that we have reduced transport means that the largest stabilizing and destabilizing terms are almost balancing so that small effects become important. The corresponding thing happens with MHD type modes, i.e. the physics description becomes important close to marginal stability. 
Another example of effect of the physics description on drift waves is the growth of transport coefficients with radius.

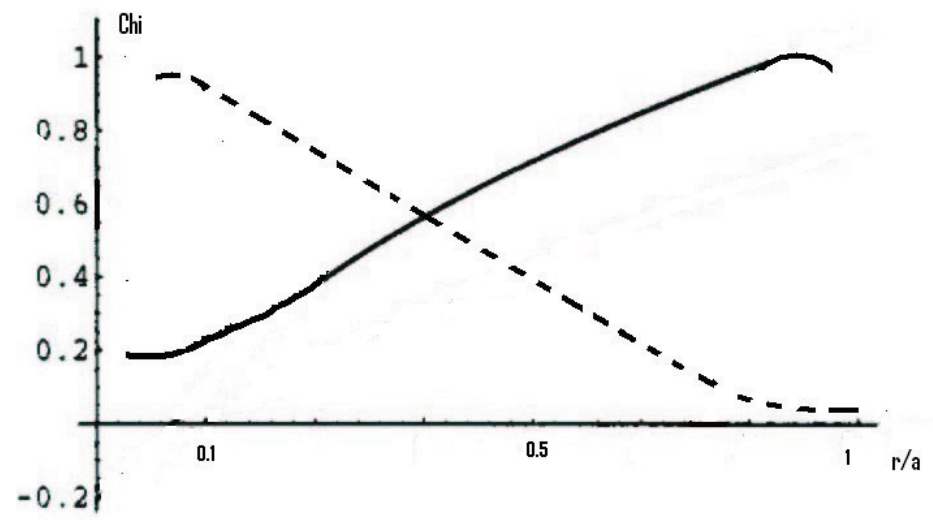

FIGURE 5. Radial profiles of $\chi$ for a fluid or kinetc model that is expanded in $\omega \mathrm{D} / \omega$ (dashed) and an unexpanded model (full line). The experimental curve is also close to the full line.

It is instructive to look at the linear gyrokinetic density perturbation for ions.

$$
\frac{\delta \mathrm{n}_{\mathrm{i}}}{\mathrm{n}_{\mathrm{i}}}=-\frac{e \phi}{T_{i}}\left[1-\frac{1}{n_{0}} \int_{0}^{\infty} \frac{\omega-\omega_{* i}\left[1+\eta_{i}\left(m_{i} \mathrm{v}^{2} / 2 T_{i}-3 / 2\right]\right.}{\omega-\omega_{D_{i}}\left(\mathrm{v}_{\|}^{2}+\mathrm{v}_{\perp}^{2} / 2\right) / \mathrm{v}_{\mathrm{th}}^{2}} J_{0}(\xi)^{2} f_{0} d^{3} \mathrm{v}\right]
$$

We have already shown an expansion of this expression (1.24) but that was just in order to explore the similarity to the fluid response. The question is now under what circumstances we may be allowed to expand (1.30) in $\omega_{\mathrm{D}} / \omega$. If (1.30) for ions is combined with the Boltzmann response for electrons, the drift frequencies are the only frequencies that can generate $\omega$. Thus we have to assume that $\omega$ will be of the order of the drift frequencies. Now we want to expand in $\omega_{\mathrm{D}}$ so $\omega *$ should be larger. Then assuming $\omega$ to be of order $\omega *$, the critical parameter to expand in is

$$
\varepsilon_{n}=\frac{\omega_{D}}{\omega_{*}}=2 \frac{L_{n}}{L_{B}}
$$

where $L_{B} \sim R$. In practice $\varepsilon_{n}$ is typically of order 1 in the core and at least out to $\mathrm{r} / \mathrm{a}$ $=0.8$. Close to the axis $\varepsilon_{\mathrm{n}}$ goes to infinity. Using (1.13) and Bolzmann electrons we arrive at:

$$
\chi_{i}=\frac{1}{\eta_{i}}\left(\eta_{i}-\frac{2}{3}-\frac{10}{9 \tau} \varepsilon_{n}\right) \frac{\gamma^{3} / k_{r}^{2}}{\left(\omega_{r}-\frac{5}{3} \omega_{D i}\right)^{2}+\gamma^{2}}
$$

It is obvious that (1.32) leads to the type of thermal conductivity shown in Fig 6 for the unexpanded model at least close to the axis since both $\mathrm{L}_{\mathrm{n}}$ and $\mathrm{L}_{\mathrm{T}}$ become large towards the axis so that $\eta$ remains finite. As it turns out, we need also electron trapping to recover the full curve in Fig 3. Both $\varepsilon_{n}$ and $\omega_{D}$ in (1.24) are due to the curvature effect of the diamagnetic heatflow as given by (1.12). Thus the growth of 
$\chi$ with radius in Fig (6) is due to both magnetic curvature and nonadiabaticity. Now, $\mathrm{Eq}(1.13)$ is directly connected to the fluid closure since at marginal stability:

$$
\omega_{r}=\frac{5}{3} \omega_{D_{i}}
$$

so we are at the fluid resonance at marginal stability. However, this works well, both in comparison with kinetic theory and experiment. Fig 6 compares qualitatively and semi- quantitatively the linear growth, nonlinear saturation and continued oscillations for a fluid model with nonlinear closure and a reactive fluid model according to Ref 13 and the Hammett Perkins Gyro - Landau fluid model according to Ref 12 ( Mattor and Parker). The interaction is between two slab ITG modes and a zonal flow.

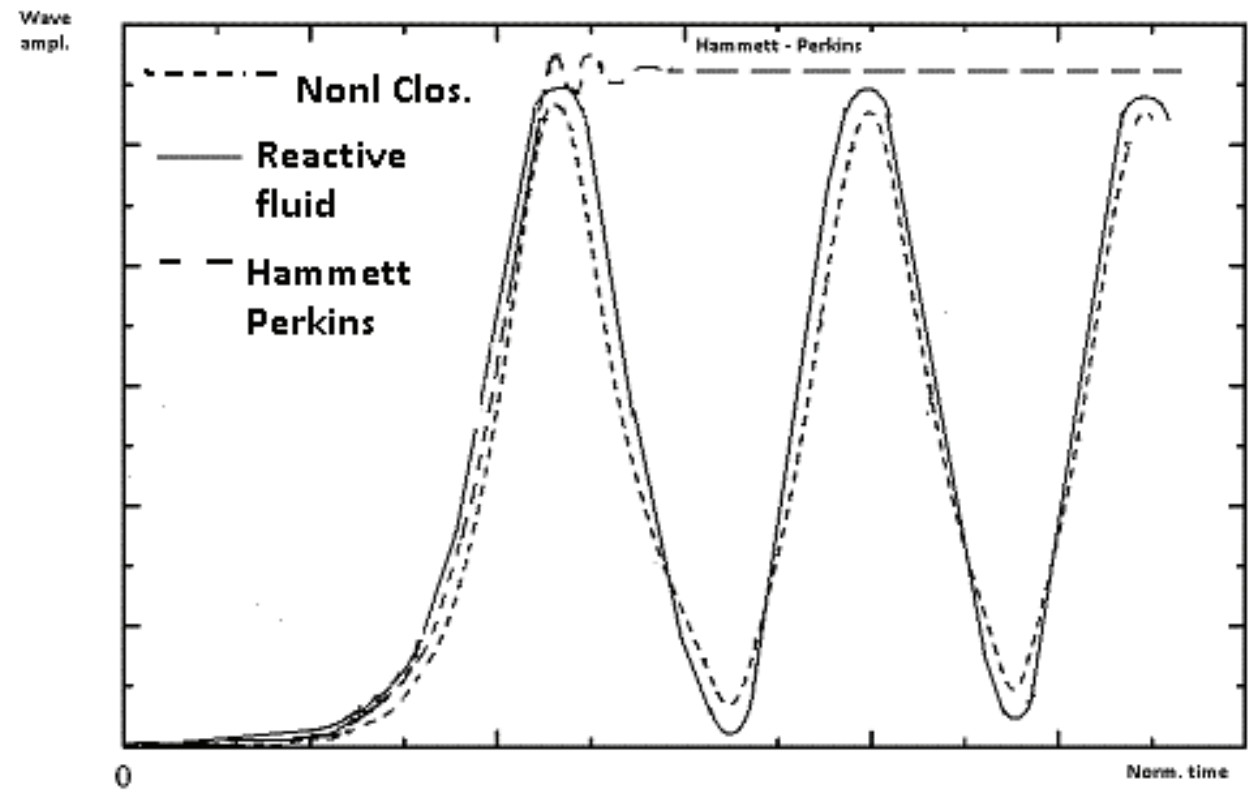

FIGURE 6. Development in time of three-wave interaction between two slab ITG modes and a zonal flow with different fluid descriptions including reactive fluid, fluid with nonlinear closure and the Hammett Perkins gyro-Landau fluid model.

The paper by Mattor and Parker showed that the fluid model with a nonlinear closure is quite close to the full kinetic model. The only difference between the reactive model and the model with nonlinear closure is the kinetic resonance. The velocity distribution is here Maxwellian and the highest moment (fifth) is expressed through the kinetic integral where a nonlinear frequency shift is included, i.e.

$$
\omega=\omega_{L}+\delta \omega_{n l}
$$


The result of the nonlinear frequency shift in the kinetic integral may be difficult to see by inspection. However, in the expanded form, corresponding to the Universal instability (driven by inverse Landaudamping) it can be visualized.

$$
\gamma=\left(\frac{\pi}{2}\right)^{1 / 2} \omega_{* e} \frac{\omega-\omega_{* e}}{k_{\|} v_{t e}} e^{-\omega^{2} /\left(k_{\|} v_{t e}\right)}
$$

As seen from (1.35), the dissipation (energy) changes sign when the frequency equals the diamagnetic drift frequency. Thus the nonlinear frequency shift could easily change the sign of the imaginary part $\gamma$. This is what happens in Fig 7. As can be seen the wave particle resonance is stabilizing near maxima and destabilizing near minima. It is the absence of this effect that causes the Hammett Perkins model to phase lock at an amplitude above the other models. Thus kinetic resonances effectively vanish although we keep the Maxwellian distribution function. This particular case is coherent but turbulence can be seen as due to coupling of very many such systems. Already two waves lead to stochasticity of marginally trapped - detrapped particles ${ }^{15}$. (Chirikov) Stochastic particles diffuse quasilinearly. However, in practice nonlinear effects reintroduce correlations. The first example of this is the kinetic equation for waves, the Random phase approximation Ref 16.

The next example is the nonlinear Fokker Planck equation:

$$
\left(\frac{\partial}{\partial t}+\mathrm{v} \frac{\partial}{\partial x}\right) f(x, \mathrm{v}, t)=\frac{\partial}{\partial \mathrm{v}}\left[\beta \mathrm{v}+D^{\mathrm{v}} \frac{\partial}{\partial \mathrm{v}}\right] f(x, \mathrm{v}, t)
$$

When we consider turbulent collisions we are in a random phase situation where the friction coefficient $\beta$ and diffusivity in velocity space $D^{v}$ are proportional to sums of intensities of wave amplitudes (phase dependent terms have been averaged out).

Clearly friction in this case gives a nonlinear frequency shift which is a strongly nonlinear feature, i.e. nonlinearities have reintroduced correlations. When the coefficient are constants, (1.36) has an analytical solution ${ }^{14}$ (S. Chandrasekhar). The solution has the shape shown in Fig 8.

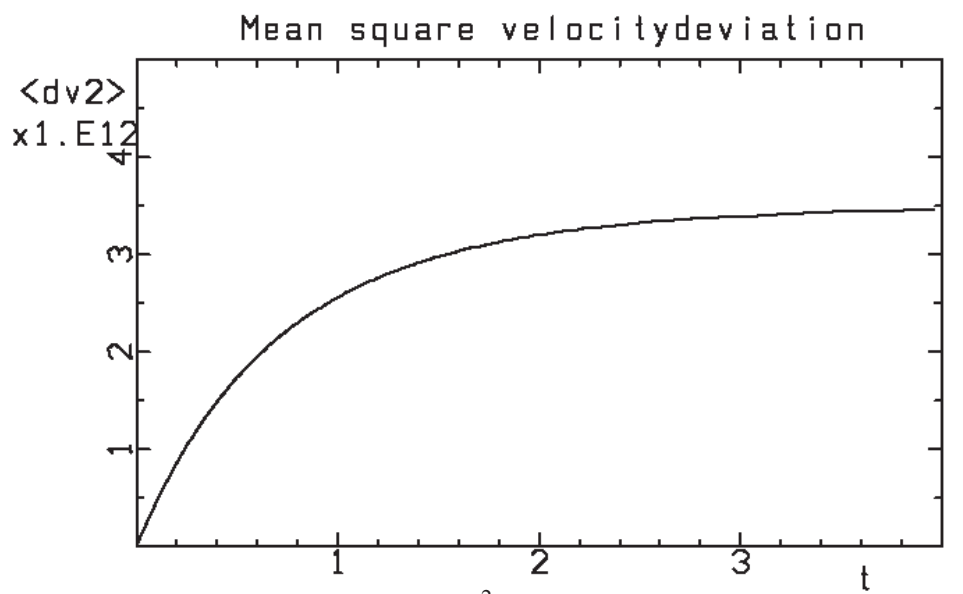

FIGURE 7. Mean square velocitydeviation $\left\langle(\Delta v)^{2}>\right.$ as a function of time showing intitial quasilinear linear growth and later saturation due to strongly nonlinear effects. 
Here the first linearly growing part corresponds to quasilinear diffusion while the asymptotic flat part is strongly nonlinear. Thus the quasilinear part represents completely random behavior while the flattening is due to correlations introduced my nonlinear effects. This type of behavior can be obtained by renormalization ${ }^{17,18}$. An important aspect of the flat part is that there is no energy transfer between resonant particles and waves on the average. Thus linear wave-particle resonances have been averaged out! This is analogous to the coherent case we just discussed. In both cases the phase mixing of linear resonances is due to strongly nonlinear effects.

A sensitive test on the presence of linear kinetic resonances is the strength of particle pinches $^{22}$. In Fig 8 we show Chi and $\mathrm{D}$ as a function of temperature gradient for a reactive fluid model, and a fluid model with Landau damping.

Fig $8 \mathrm{a}$

Fig 8c
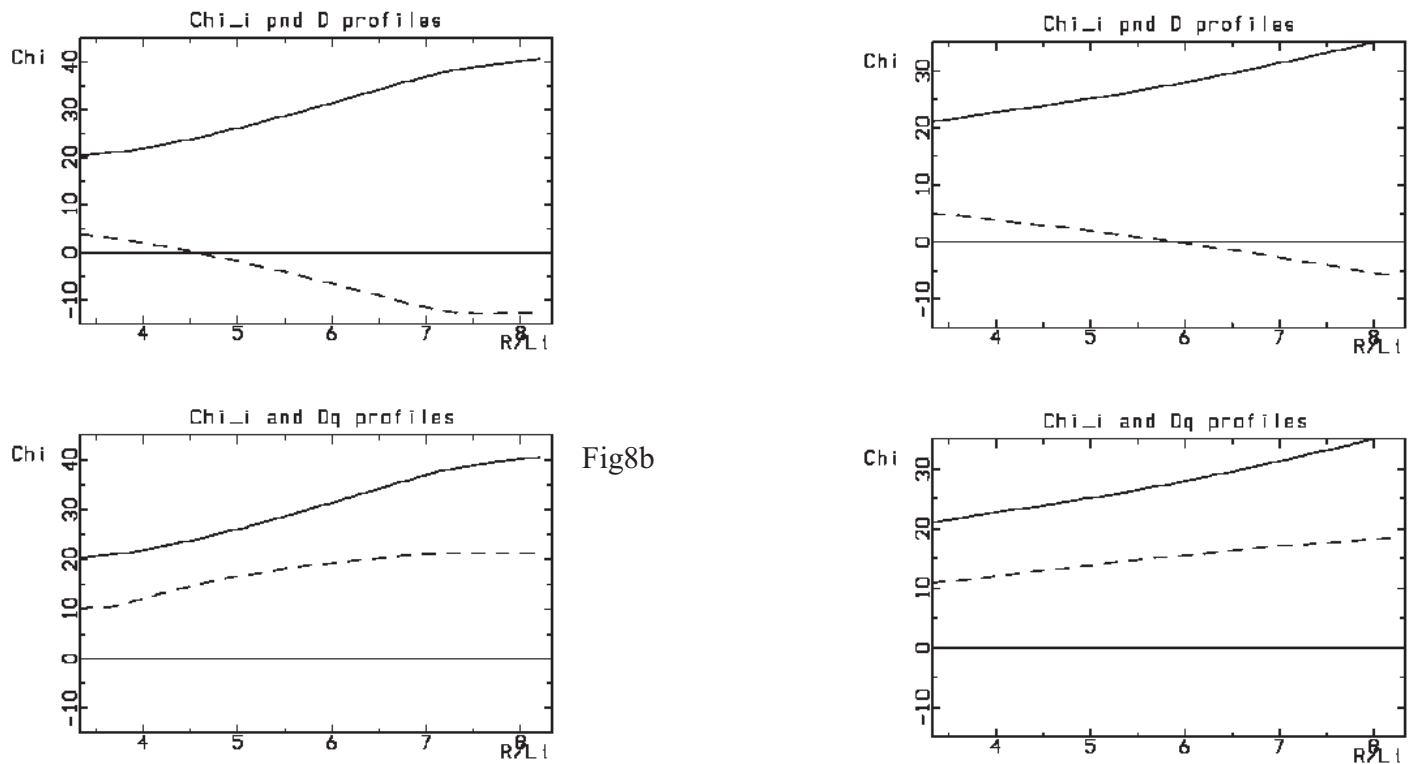

Fig8b

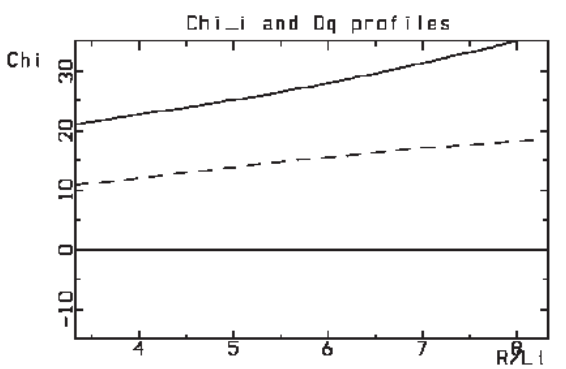

Fig $8 d$

FIGURE 8. Particle transport as a function of temperature gradient for a reactive fluid model a) and b) and for a fluid model where Landaudamping was added, c) and d). The full lines show $\chi_{i}$ for comparison while the dotted lines show particle diffusivities. Here a) and c) show that of the main ions

(Hydrogen) while b) and d) show the diffusivities of Coal. From Ref 22 with the permission of the American Institute of Physics. 
Fig 9 shows the quasilinear particle diffusion for the same parameters.

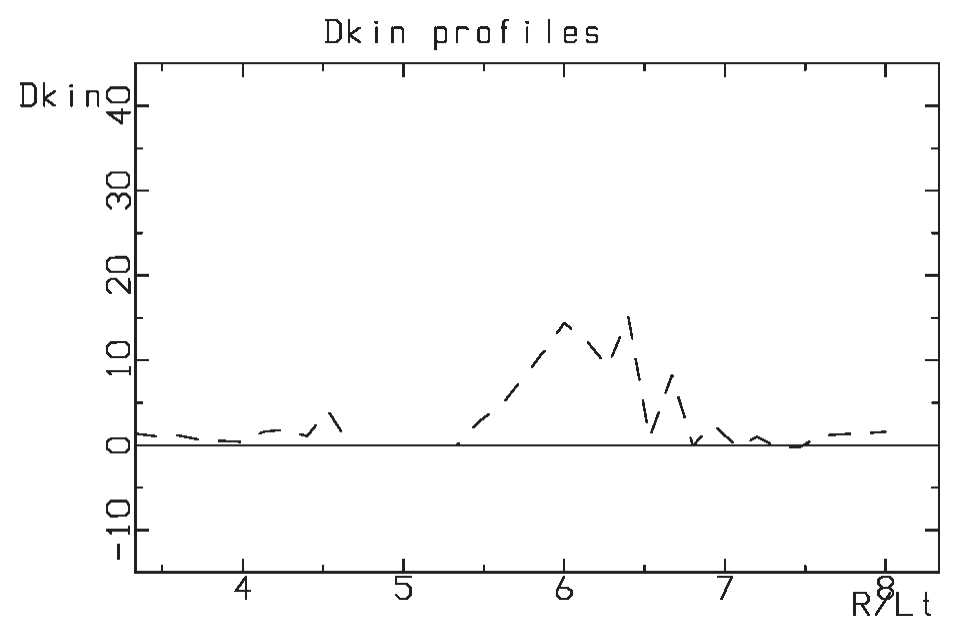

FIGURE 9. Particle diffusivity in Quasilinear kinetic theory for the same parameters as in Fig $4 . \mathrm{k}_{\theta} \rho_{\mathrm{s}}$ $=0.3$ From Ref 22 with the permission of the American Institute of Physics.

We note that Landau damping reduces the particle pinch. In the case shown in Fig 8 Landau damping is introduced in the energy equation while it is present for all moments in the quasilinear case. Thus the effect is stronger in the quaslininear case. In these examples we used the typical mode number

$$
k_{\theta} \rho_{s}=0.3
$$

As it turns out, the existence of the quasilinear particle pinch depends on modenumber. However, in order to obtain the total transport, we need to consider a modenumber corresponding to the inverse correlation length of the system. This is typically given by (1.37) but can vary due to different parameters, typically due to magnetic shear, flowshear and magnetic q. This variation would usually be between 0.2 and 0.4 .

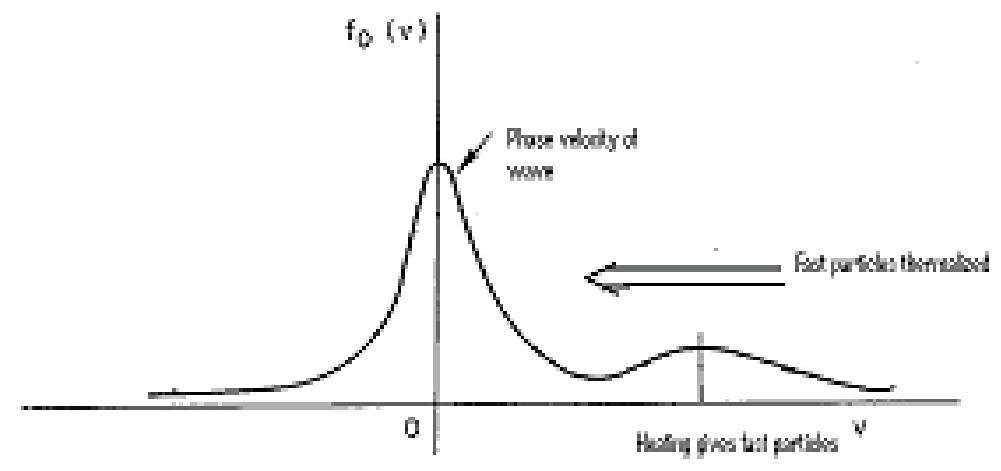

FIGURE 10. Separation in phase velocity between drift waves and heating. 


\section{TIME SCALES}

Since the confinement time is limited in a reactor, we need to reach sufficiently high reaction rate to get out more energy than we put in during a confinement time. Since we, in a tokamak, have a pulsed operation, the pulse time is the time we need to control the plasma. It is instructive to compare the different timescales in a reactor as shown in Fig 11.

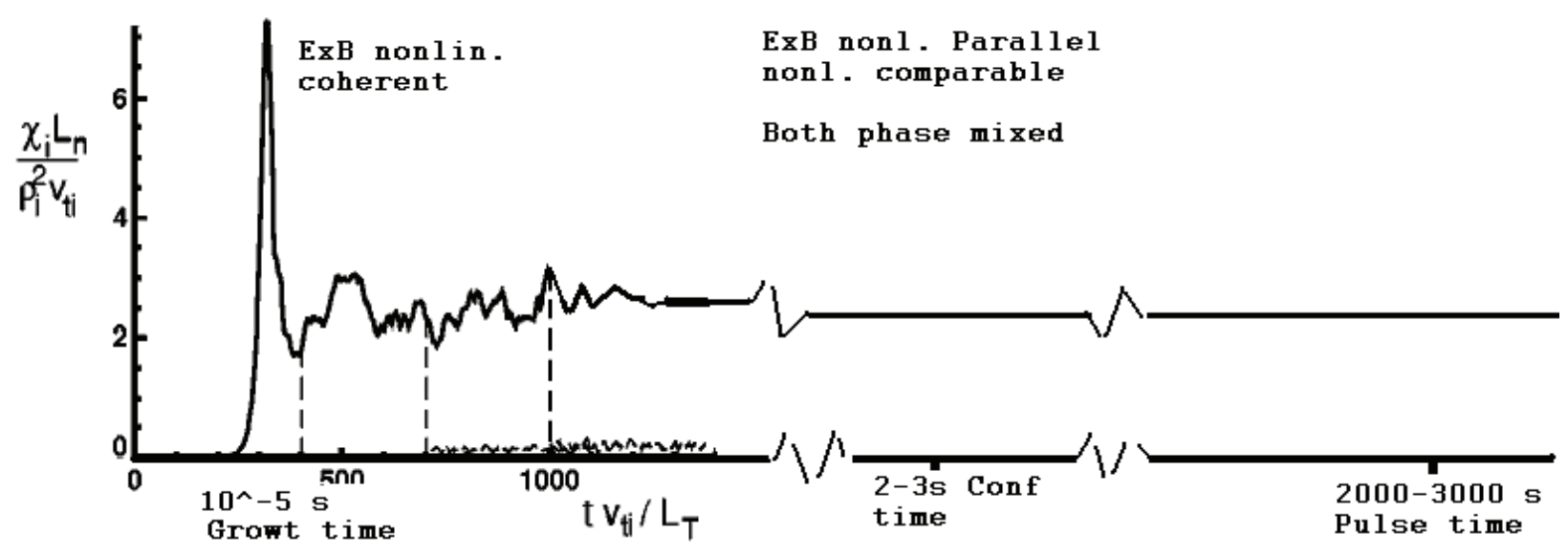

FIGURE 11. Timescales in a fusion reactor

While the growth time is a few times $10^{\wedge}-5$, the confinement time is typically $2-3 \mathrm{~s}$. A quasistationary turbulent spectrum appears to exist after about $10^{\wedge}-3 \mathrm{~s}$. A lot of discussions have concerned the relevance of the parallel nonlinearity. Several aspects were given in Ref 23 , primarily dealing with the relevance of the parallel nonlinearity. There it was pointed out that the parallel nonlinearity enters on the transport timescale. Since the main parallel nonlinearity is due to Nonlinear Landaudamping ${ }^{24}$, which considers beating of waves with almost equal modenumbers, we conclude that this nonlinearity can be seen as nonlinear Landaudamping. The fact that the parallel nonlinearity enters on the confinement timescale was also noted in Ref 25. This is, actually, also true for the nonlinear frequency shift in the Fokker-Planck equation. As a result of this ordering, also the friction and diffusivity in the Fokker-Planck equation enter on the confinement timescale.

Thus in order to reach steady state in kinetic simulations we, in principle, have to rum our codes on the confinement timescale.

As it turns out, most gyrokinetic simulations appear to reach steady state on a much shorter timescale. We can actually see a possibility for that from Fig 6. There the kinetic resonance is averaged out on a few growth times. However, that system is coherent while tokamak turbulence is almost completely incoherent. Thus the possibility we can see is if the kinetic resonances are averaged out due to the much 
stronger ExB nonlinearity while the dynamics is still coherent. Since there is only one resonance (1.38), particles can be taken out of resonance by either parallel or perpendicular acceleration.

$$
\omega=k_{\|} \mathrm{v}_{\|}+\omega_{D}
$$

Thus if the stronger perpendicular resonance takes particles out of resonance in the coherent phase of the development, there will be no kinetic effect of the parallel nonlinearity. Nonlinear parallel fluid effects are usually ignorable.

\section{SUMMARY}

We have here discussed one fluid, multi fluid and kinetic descriptions of fusion plasmas. While the one fluid description is of a theoretical interest for comparison with ordinary fluids (Navier Stokes equation) and gives a simple and elegant description of the fast, global ideal MHD instabilities, it can usually be replaced by a multi fluid description with minimal extra effort in the area of its application. When a multi fluid description is required, the physics is more complicated and this, of course, has to show in the complexity of the description. The kinetic description can often be made elegant but, of course, the necessity to follow many particle orbits make calculations heavy. A fluid description also uses intuitively clear pictures in terms of density, temperature etc. As we have seen here a fluid description including all moments with sources in the experiment has to emerge as the full description on the confinement timescale.

\section{REFERENCES}

1. H. Alfvén, Existence of electromagnetic-hydrodynamic waves, Nature 3, October 1942, p405.

2. I.B. Bernstein, E.A. Frieman, M.D. Kruskal and R.M. Kulsrud, An Energy Principle for Hydromagnetic Stability Problems, Proc. Royal Society of London A244, 17 (1958).

3. G. Bateman, MHD Instabilities, MIT Press, Cambridge Massachusetts 1978.

4. B. Lehnert, Dynamics of Charged Particles, North-Holland, Amsterdam 1964.

5. J. Weiland, Collective modes in Inhomogeneous Plasmas, Kinetic and Advanced Fluid Models.

6. J. Weiland and H. Nordman, Theory of Fusion Plasmas, Proc. Varenna-Lausanne Workshop (Chexbres 1988) p 4511988.

7. A. Zagorodny, J. Weiland. Phys. Plasmas 6, 2359 (1999).

8. I. Holod, A. Zagorodny, J. Weiland. Phys.Rev. E71, 046401 (2005).

9. T.H.Dupree, Physics of Fluids 10, 1049 (1967).

10. Yu.L. Klimontovich, Statistical Theory of Nonequilibrium Processes in Plasma, Pergamon Press, Oxford 1967.

11. A Hasegawa and K. Mima, Phys. Fluids 21, 87 (1978).

12. N. Mattor and S. Parker, Phys. Rev. Lett. 79, 3419 (1997).

13. I. Holod, J. Weiland and A. Zagorodny, Phys. Plasmas 9, 1217 (2002).

14. S. Chandrasekhar, Stochastic Problems in Physics and Astronomy, Rev. Modern Physics 15, 1 (1943).

15. B.V. Chirikov, Phys. Rep. 52, 263 (1979). 
16. R.Z. Sagdeev and A.A. Galeev, Nonlinear Plasma Theory, Benjamin, New York 1969.

17. T.H. Dupree, Phys. Fluids 9, 1773 (1966).

18. J. Weinstock, Phys. Fluids 12, 1045 (1969).

19. J. Weiland, A. Jarme'n and H. Nordman, Nuclear Fusion 29, 1810 (1989).

20. A. Zagorodny and J. Weiland, Physics of Plasmas 6, 2359 (1999).

21. I. Holod, A. Zagorodny and J. Weiland, Phys. Rev. E71 046401 (2005).

22. J. Weiland, A. Zagorodny and V. Zasenko, Proc. IFP-CNR - Chalmers workshop on Nonlinear Phenomena in Fusion Plasmas, AIP Proceedings 1392, 33, (2011).

23. J. Candy, R.E. Waltz, S.E. Parker and Y. Chen, Physics of Plasmas 13, 074501 (2006).

24. N.L. Shatashvili and N.L. Tsintsadze, Physica Scripta T2, 511 (1982).

25. J. Weiland, A. Eriksson, H.Nordman and A. Zagorodny, PPCF 49, 1 (2007). 